\title{
Grandezas físicas unidimensionais
}

\author{
One-dimensional physical quantities
}

\author{
G. F. Vasconcelos Júnior ${ }^{1}$, R. P. S. Costa ${ }^{1}$, B. F. Rizzuti*1 \\ ${ }^{1}$ Universidade Federal de Juiz de Fora, Departamento de Física, Juiz de Fora, MG, Brasil
}

\begin{abstract}
Recebido em 15 de Setembro, 2017. Revisado em 23 de Novembro, 2017. Aceito em 28 de Novembro, 2017.
No presente trabalho, apresentamos definições operacionais para as grandezas físicas distância, massa e tempo. Tentativas de formular uma definição operacional bem fundamentada para grandezas físicas foram primeiramente propostas no fim do século 19 por Hermann von Helmholtz e Otto Hölder. O problema consiste em como associar um número (o valor da grandeza) com uma entidade não-numérica (a própria grandeza). O trabalho apresenta uma releitura moderna dos autores mencionados. Parafraseando Feynman no seu clássico trabalho das integrais de caminho, "there is a pleasure in recognizing old things from a new point of view".
\end{abstract}

Palavras-chave: grandezas físicas, teoria da medida, definição operacional.

In the present paper, we present operational definitions of the physical quantities distance, mass and time. Attempts to formulate a well-based operational definition of physical entities were first proposed in the end of the 19-th century by Hermann von Helmholtz and Otto Hölder. The problem consists in finding how to associate a number (the value of the entity) with a non-numerical entity (the entity itself). The paper presents a modern reading of the above mentioned authors. Paraphrasing Feynman in his classic work on path integrals, "there is a pleasure in recognizing old things from a new point of view".

Keywords: physical quantities, measurement theory, operational definition.

\section{Introdução}

Em uma conversa usual e informal entre duas pessoas, é comum o uso de exemplos para explicar o significado de um objeto, procedimento ou o que quer que seja. Se a mesma conversa acontecesse entre dois matemáticos, então eles (provavelmente) evitariam o uso de exemplos e lançariam mão do que chamamos de definição, no lugar dos exemplos. Extrapolando um pouco mais e supondo que dois físicos conversassem, seria razoável esperar que no lugar de exemplos ou definições, eles usassem o que chamaremos de definição operacional. Este termo foi cunhado pelo físico P. W. Bridgman [1] e significa que nenhum conceito pode ser usado, o qual não possa ser definido a partir de um procedimento operacional. Vamos então tratar neste trabalho, seguindo este ideal, sobre a construção de grandezas físicas, à la $\mathrm{P}$. W. Bridgman.

As primeiras tentativas de se construir a noção de valores de grandezas associadas aos fundamentos de medidas foram feitas por Hermann von Helmholtz [2]. A formalização deste processo, que consiste em associar injetivamente uma estrutura qualitativa com uma estrutura algébrica (numérica), pode ser encontrada no antigo trabalho de O. Hölder [3], onde ele propõe uma teoria axiomática da medida. Uma leitura mais moderna pode ser vista em [4] e [5]. No texto acima, a palavra "associar" consiste no processo de medida; "estrutura qualitativa"

*Endereço de correspondência: brunorizzuti@fisica.ufjf.br corresponde ao conjunto de objetos para o qual a grandeza está definida e, por fim, à "estrutura numérica" associamos a quantificação da grandeza em questão. Como veremos, o conjunto de objetos para qual determinada grandeza está bem definida será dividido em classes, e é natural esperar que elementos de classes distintas possuam valores distintos da grandeza correspondente. Daí exigirmos que tal associação seja injetiva.

Para as origens históricas do que se chama tanto teoria clássica, quanto teoria representacional da medida, indicamos a referência [6]. Este artigo é uma extensão natural destes trabalhos antigos. Em um certo sentido, unimos ideias tanto de Helmholtz quanto de Hölder. Com efeito, em [2], duas perguntas podem ser identificadas, a saber

(1) Qual o significado objetivo para considerarmos dois objetos iguais sob algum critério? (2) Qual propriedade que a combinação física de dois objetos deve exibir para que possamos associar algum atributo dos objetos aditivamente e, assim, considerar este atributo a quantidade que pode ser expressa por um número concreto?

que são respondidas ao longo do nosso texto. A primeira caracterizará classes de equivalência do que chamaremos de domínio de uma grandeza física. A segunda, por sua vez, está ligada à definição de uma soma em um espaço de valores de certa grandeza. Hölder, por sua vez, apresenta 
uma estrutura matematicamente formal, fixando a razão do valor de duas grandezas como um número real. Em um certo sentido, isto está ligado com a nossa construção, uma vez que a razão $[a: b]=r \in \mathbb{R}$, como Hölder a define, é equivalente à nossa definição de multiplicação do valor de uma grandeza por um escalar, como será visto na expressão 10. Desta maneira, este artigo é uma extensão natural destes trabalhos clássicos, por usarmos o formalismo da Álgebra Linear para caracterizarmos o que é definido um pouco à frente como espaço de valores. Além disso, construímos em detalhes exemplos para ilustrar todo este formalismo moderno, que pode ser visto em [5].

A tentativa de se definir o que é uma grandeza pode ser vista no Joint Committee for Guides in Metrology (JCGM) [7:

property of a phenomenon, body, or substance, where the property has a magnitude that can be expressed as a number and a reference.

Esta definição tem algumas restrições que passamos a enumerar.

1-O que magnitude quer dizer?

2-Como comparar grandezas de magnitudes diferentes? 3-O que é um número neste contexto?

4-Assumindo certo conhecimento prévio, intuímos que referência quer dizer a unidade de medida da grandeza. Qual o seu significado?

5-É possível exibir uma lista infindável de grandezas físicas que não podem ser descritas com a definição do JCGM: força, velocidade, etc; basta que a grandeza seja vetorial ou tensorial.

O texto do JCGM fica ainda vago em outro ponto que será fortemente discutido aqui. Uma medida corresponde a:

process of experimentally obtaining one or more quantity values that can reasonably be attributed to a quantity.

Curiosamente veremos que o processo de medida, em um certo sentido, sequer precisa fornecer um valor numérico para a grandeza correspondente. O processo está, na verdade, ligado com a caracterização de objetos que possuem o mesmo valor de determinada grandeza, mesmo sem conhecermos o valor de tal grandeza.

Fornecer a noção de uma grandeza física somente com uma definição como a da JCGM, acima, pode tornar confuso e vago o entendimento de tal noção. Desta maneira, o conceito de grandeza será construído ao longo deste artigo. Discutiremos como exemplo as três grandezas que constituem os pilares da mecânica clássica: distância, massa e tempo, que eventualmente serão denotadas por L, M e T, respectivamente. Sempre que possível, daremos exemplos para motivar as construções que serão tratadas. O trabalho será dividido da seguinte maneira: na Seção II, apresentamos as definições iniciais que caracterizam uma grandeza: seu domínio, espaço de valores e para elucidar esta construção inicial, detalhamos como são construídas as grandezas L, M e T. Na Seção III estendemos a discussão anterior com uma lista de comentários, em forma de subseções, e mostramos como podem ser definidas a multiplicação e divisão entre grandezas. Um comentário razoavelmente extenso sobre incertezas experimentais também pode ser visto. Por fim, a Seção IV é deixada para as conclusões.

\section{Grandezas físicas}

Comecemos então com uma grandeza física que chamaremos de $G$. A primeira coisa que precisamos para caracterizá-la é definir o conjunto para o qual $G$ faz sentido. Chamaremos este conjunto de domínio de $G$ e ele será representado por $D_{G}$. Vejamos agora como é importante construir o domínio de determinada grandeza. A pergunta "Qual a temperatura da obra Dom Quixote, de Miguel de Cervantes?" simplesmente não faz sentido. Com efeito, a grandeza física temperatura não pode ser atribuída a um trabalho literário. Fixado o domínio de $G$, definimos em $D_{G}$ uma relação de equivalência, a partir de um procedimento experimental de comparação, que acaba por caracterizar objetos que terão o que chamaremos de mesmo valor da grandeza $G$. Curiosamente a cognição humana busca padrões e acaba classificando objetos como semelhantes ou distintos, seja organizando talheres em uma gaveta, conforme a Fig. 1, ou fazendo taxonomia biológica. Aproveitando o exemplo dos talheres, vamos formalizar o conceito de classes de equivalência, que será utilizado em larga escala ao longo deste trabalho. Seja $D$ um conjunto. Definimos uma relação como um subconjunto do produto cartesiano $D \times D$. Os elementos de $\sim \subset D \times D$ são denotados por $d_{1} \sim d_{2}$, com $d_{1}, d_{2} \in D$. Quando uma relação $\sim$ é reflexiva, simétrica e transitiva, isto é,

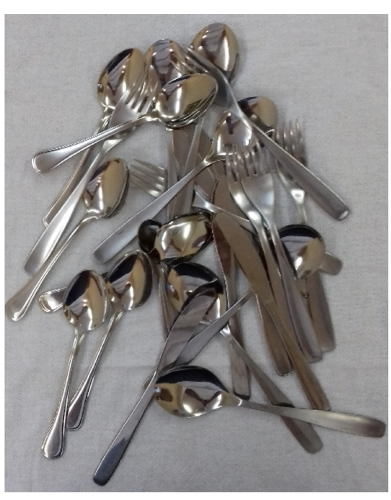

(a)

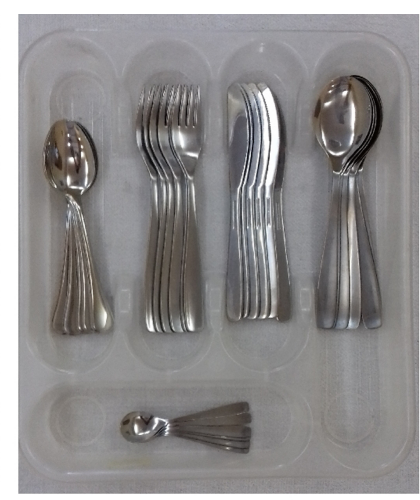

(b)
Figura 1: (a) mostra um conjunto de talheres e (b) representa o mesmo conjunto organizado em classes de equivalência de tipos de talheres. 


$$
\begin{gathered}
\text { i) } d \sim d, \forall d \in D, \\
\text { ii) } d_{1} \sim d_{2} \Rightarrow d_{2} \sim d_{1}, \forall d_{1}, d_{2} \in D, \\
\text { iii) } d_{1} \sim d_{2} \text { e } d_{2} \sim d_{3} \Rightarrow d_{1} \sim d_{3}, \forall d_{1}, d_{2}, d_{3} \in D,
\end{gathered}
$$

então é chamada de uma relação de equivalência. Dado um elemento $d \in D$, definimos a classe de equivalência $[d]$ como o seguinte subconjunto de $D$,

$$
[d]=\{x \in D ; x \sim d\} .
$$

A importância para a "organização" de um conjunto reside em três propriedades associadas com as classes $[d] \subset D$

$$
\begin{aligned}
& \text { p1) } d_{1} \sim d_{2} \Rightarrow\left[d_{1}\right]=\left[d_{2}\right], \\
& \text { p2) } d_{1} \nsim d_{2} \Rightarrow\left[d_{1}\right] \cap\left[d_{2}\right]=\phi, \\
&p 3) D=\bigcup_{d \in D}[d] .
\end{aligned}
$$

As três propriedades acima são bem retratados pelos talheres: a classe de garfos pode ser representada por qualquer garfo (p1). Como colher e faca não se relacionam, a interseção entre o subconjunto de colheres e o de facas é vazia (p2). Por fim, unindo todas as classes de garfos, facas, colheres, etc, obtemos todo o conjunto de talheres (p3). Toda esta exposição motiva definirmos a partição de $D{ }^{1}$

$$
D / \sim=\{[d] ; d \in D\},
$$

formada por classes de equivalência $[d] \subset D$.

A relação de equivalência fornece um procedimento experimental para determinação de objetos do mesmo tipo em $D_{G}$, que organiza o domínio da grandeza em classes de equivalência. Ressaltamos que objetos distintos podem ser equivalentes quanto a uma grandeza e não equivalentes em relação a outra (cubos sólidos de materiais distintos podem ser equivalentes quanto aos volumes mas não equivalentes quanto às massas).

Consideremos um exemplo. Em uma aula de ondas sonoras e cordas vibrantes, o professor decide mostrar que uma corda de um violão emite a mesma nota, só que com uma oitava acima, bastando para isso diminuir o tamanho da corda pela metade. Sem conhecer o tamanho da corda, o professor usa um fio inextensível para medir o comprimento da corda e a dobra no meio. O fio inextensível, partindo de um dos lados onde a corda está fixa, atinge exatamente a décima segunda casa do braço do violão, coincidindo com a prometida oitava acima. É notável que o professor tenha feito uma medida de uma distância sem ter em mãos uma fita métrica ou régua.

\footnotetext{
${ }^{1}$ Uma partição de um conjunto $D$ é uma coleção disjunta de subconjuntos não vazios de $D$, cuja união é $D$ 8.
}

Aproveitando o exemplo do tamanho do fio inextensível, gostaríamos de associar valores numéricos às classes de equivalência em $D_{G}$. Vamos chamar o conjunto de valores que caracterizam uma classe de $V_{G}$. Se necessário, podemos estender $V_{G}$ para garantir uma estrutura matemática boa o suficiente. E que estrutura é esta? Antes de responder esta pergunta, já mencionamos que dois objetos $g$ e $h$ em $D_{G}$ possuem o mesmo valor da grandeza $G$ $\left(v_{g}=v_{h} \in V_{G}\right)$, se, e somente se, $g \sim h$, isto é, $g$ e $h$ são equivalentes. Para descobrir a estrutura de $V_{G}$, consideremos mais dois exemplos. Colocamos sobre uma balança dois objetos distintos, digamos um telefone celular e um livro. A balança simplesmente indica a massa que foi colocada sobre sua plataforma, sem fazer distinção se colocamos um ou mais objetos. Com este exemplo somos induzidos a definir uma soma,

$$
+: V_{G} \times V_{G} \longrightarrow V_{G}
$$

onde $\times$ denota o produto cartesiano.

Passemos agora ao segundo exemplo, que trata de um fio inextensível totalmente esticado usado para medir comprimentos no braço do violão. Se associarmos um valor $v_{L}$ ao fio, então, naturalmente a metade do braço mediria $\frac{1}{2} v_{L}$. Esta ideia pode ser estendida para obtermos valores de comprimentos na forma $q v_{L} \operatorname{com} q$ sendo um número racional positivo. Para reproduzir uma estrutura matemática conhecida, vamos definir, no caso geral, em $V_{G}$, o produto por números reais,

$$
\cdot: \mathbb{R} \times V_{G} \longrightarrow V_{G} \cdot
$$

Poderíamos exigir certas propriedades da soma (9) e do produto por números 10 e tomá-los como axiomas. Mas, seguindo a prescrição de P. W. Bridgman, de construir a noção de grandezas físicas a partir de uma prescrição operacional, é possível verificar experimentalmente que $(90$ e (10) satisfazem as propriedades de um espaço vetorial [9] (pelo menos para os casos L, M e T que trataremos aqui, isto pode ser visto diretamente). A pergunta natural é: qual a dimensão do espaço $V_{G}$ ? Este trabalho será dedicado às grandezas cujo espaço de valores é unidimensional. Por fim, damos, assim, significado físico para as unidades utilizadas em valores de grandezas físicas: elas correspondem a uma base de $V_{G}$.

Para clarear toda esta construção, passaremos aos exemplos. Discutiremos as três grandezas principais usadas ao se desenvolver a mecânica clássica: L, M e T.

\subsection{Distância espacial}

Começamos esta subseção definindo o conjunto para o qual a grandeza distância $(L)$ está bem definida. Para elucidar os elementos do domínio $D_{L}$, considere a seguinte pergunta absurda: qual a distância da cidade? Para corrigir este questionamento, alguém poderia reestruturá-lo da seguinte maneira: Qual a distância entre as cidades A e B? Em um mapa, cidades são representadas por pontos 
e com a escala correspondente, a distância de A a B está bem definida. Assim,

$$
D_{L}=\{\text { pares de pontos }\}
$$

O procedimento experimental que define as classes de equivalência em $D_{L}$ é feito com um compasso. Colocamos uma das pernas de um compasso sobre um ponto e a outra perna é colocada sobre um segundo, sem retirar a primeira perna do primeiro ponto. Com a abertura fixada, buscamos pares de pontos que se encaixam no compasso. De acordo com a Fig. 2, diremos que $d(A, B)=d(C, D)$ e $d(A, B) \neq d(E, F)$.

Na etapa (i) o compasso é carregado do par $(A, B)$ até o par $(C, D)$ e em (ii), ele é levado até EF. A linha pontilhada corresponde a pontos $\mathrm{X}$ tal que $d(A, B)=$ $d(E, X)$.

Neste ponto cabe um comentário sobre a palavra ponto utilizada anteriormente. Ponto é tido como um conceito primitivo. A geometria euclideana não define o que são pontos, retas ou planos. São postos axiomas que relacionam os três e, somados a regras de lógica, são extraídos teoremas. Novamente adotando a postura "operacional", tanto pontos quanto retas e planos podem de fato ser definidos [5].

Agora, fixamos um comprimento padrão que define a base em $V_{L}$, que chamaremos de metro. Isto era feito no século XIX com uma barra metálica, composta por uma liga de platina com irídio, armazenada no Escritório Internacional de Pesos e Medidas em Paris de maneira a garantir que os efeitos de dilatação térmica da barra fossem mínimos. Atualmente o metro padrão é definido, de maneira mais refinada e precisa, como a unidade de comprimento igual 1.650.763,73 comprimentos de onda da radiação eletromagnética emitida pelo isótopo ${ }^{86} \mathrm{Kr}$ na sua transição entre os estados $2 p_{10}$ e $5 d_{5}$ [10].

Por fim, resta construir a soma e a multiplicação por números em $V_{L}$. Veja que a afirmação $3 \mathrm{~m}+5 \mathrm{~m}=8 \mathrm{~m}$ é vazia no seu sentido geométrico. Não sabemos quais pontos no espaço satisfazem tal igualdade ${ }^{2}$ Sejam então $(A, B)$ e $(C, D)$ dois pares de pontos; gostaríamos de

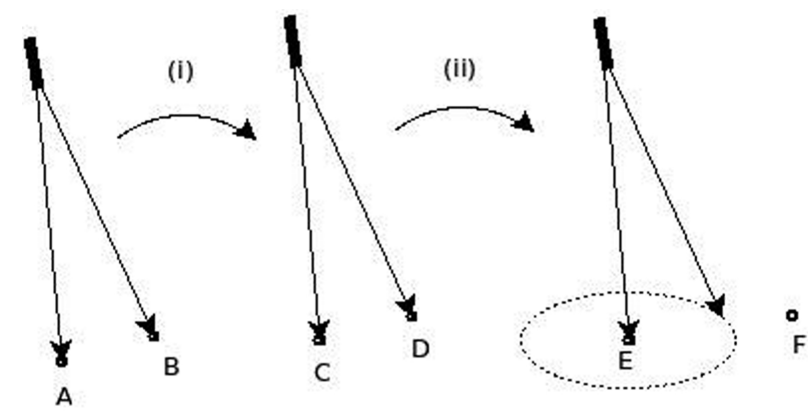

Figura 2: Procedimento de obtenção de classes de equivalência em $D_{L}$.

\footnotetext{
${ }^{2}$ A palavra 'espaço' precisa ser ancorada com uma definição operacional. Veja, por exemplo, 11 .
}

definir a soma das distâncias entre eles $d(A, B)+d(C, D)$. Fixamos um ponto $O$ e marcamos todos os pontos $X$ tal que $d(X, O)=d(A, B)$. Este conjunto é chamado de esfera com centro em $O$ e raio $d(A, B)$, que denotaremos por $\mathcal{B}(O, d(A, B))$. Escolhemos um ponto $O^{\prime}$ qualquer de $\mathcal{B}(O, d(A, B))$ e construímos $\mathcal{B}\left(O^{\prime}, d(C, D)\right)$, como na Fig. 3.

Veja que temos uma relação de ordem. Fixando uma das pernas de um compasso em $O$, buscamos pontos em $\mathcal{B}\left(O^{\prime}, d(C, D)\right)$. Quando a perna livre do compasso toca um ponto $E$ de $\mathcal{B}\left(O^{\prime}, d(C, D)\right)$ sem atingir $\mathcal{B}(O, d(A, B))$, dizemos que

$$
d(A, B)>d(O, E)
$$

Caso o ponto $F$ que a perna do compasso toca em $\mathcal{B}\left(O^{\prime}, d(C, D)\right)$ esteja sobre $\mathcal{B}(O, d(A, B))$, dizemos que

$$
d(A, B)=d(O, F) .
$$

Por fim, se a perna atravessar $\mathcal{B}(O, d(A, B))$ para atingir $\mathcal{B}\left(O^{\prime}, d(C, D)\right)$ em $G$, então

$$
d(A, B)<d(O, G)
$$

Finalmente, definimos a soma $d(A, B)+d(C, D)$ como o máximo do conjunto

$$
\left\{d(O, Y) ; Y \in \mathcal{B}\left(O^{\prime}, d(C, D)\right)\right\}
$$

É um fato experimental que o conjunto acima, Eq. 15, possui um máximo. Com esta construção, evitamos o uso da palavra reta sem uma definição e ganhamos naturalmente qual a distribuição espacial dos pontos que compõem uma soma de distâncias.

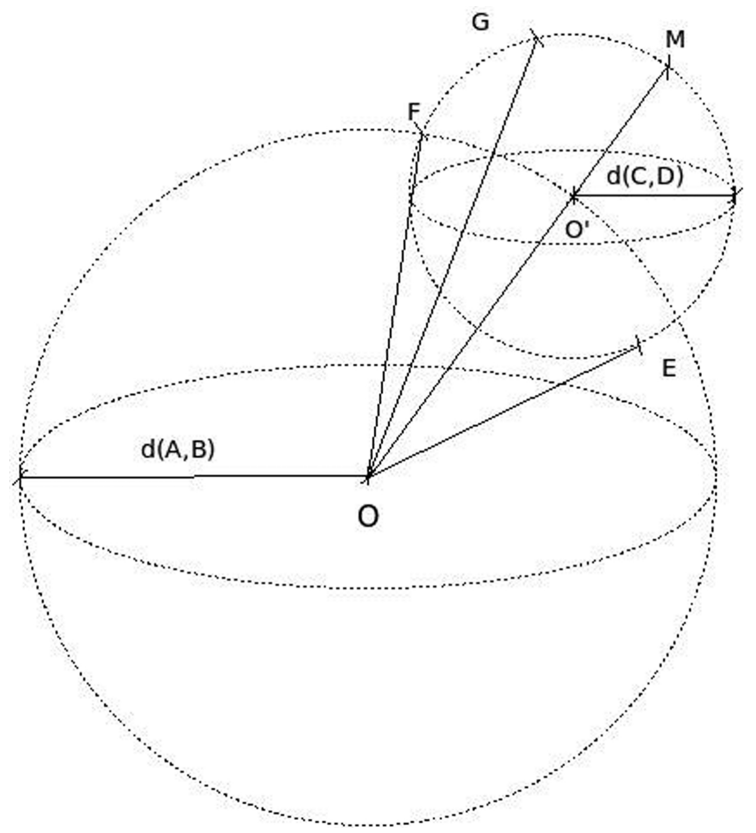

Figura 3: Obtenção experimental da soma $d(A, B)+d(C, D)$. 
A multiplicação por números em $V_{L}$ pode ser obtida à luz do exemplo com a corda de violão, que discutimos anteriormente. Fixamos uma distância com um compasso, digamos $d(A, B)$ e esticamos nosso fio inelástico por dois pontos de apoio, digamos $A_{0}$ e $A_{N}$. Ele é mantido esticado ao ser pendurado por dois pesos, conforme a Fig. 4. Marcamos então ao longo do fio pontos sucessivos com o compasso, $A_{0}, A_{1}, \ldots, A_{N}$, para algum $N$ natural. Assim, temos

$$
\begin{array}{r}
d\left(A_{0}, A_{k}\right)=k d(A, B), k=0,1, \ldots, N ; \\
d\left(A_{0}, A_{k}\right)=\frac{k}{N} d\left(A_{0}, A_{N}\right), k=0,1, \ldots, N,
\end{array}
$$

que define a multiplicação de valores de distâncias por racionais.

Infelizmente não podemos fornecer um procedimento experimental que forneça a prescrição para multiplicação de valores em $V_{L}$ por números reais. O máximo que conseguiríamos fazer seria diminuir a abertura do compasso cada vez mais. Mas temos um limite físico para tal diminuição. Invocar a densidade de $\mathbb{Q}$ em $\mathbb{R}$ é um procedimento meramente formal e não é adequado para a construção via procedimentos experimentais que propomos aqui. Um pouco mais à frente, esta discussão será ampliada; veja o comentário 3.5 ao fim da Seção III.

\subsection{Massa}

Como fizemos ao definir distância, nosso primeiro passo ao construir a noção de massa consiste em obter seu domínio. Para tal, utilizaremos o próprio aparato experimental que definirá as classes de equivalência em $D_{M}$, uma balança de dois pratos, como na Fig. 5

A barra $v$ é colocada na vertical $\}^{3}$ a barra $h$ faz um ângulo reto com $v$ e a balança ideal é aquela que permanece inalterada quando imaginamos a operação de simetria de refletir um dos lados por um espelho que contenha o eixo $v$ e seja perpendicular a $h$.

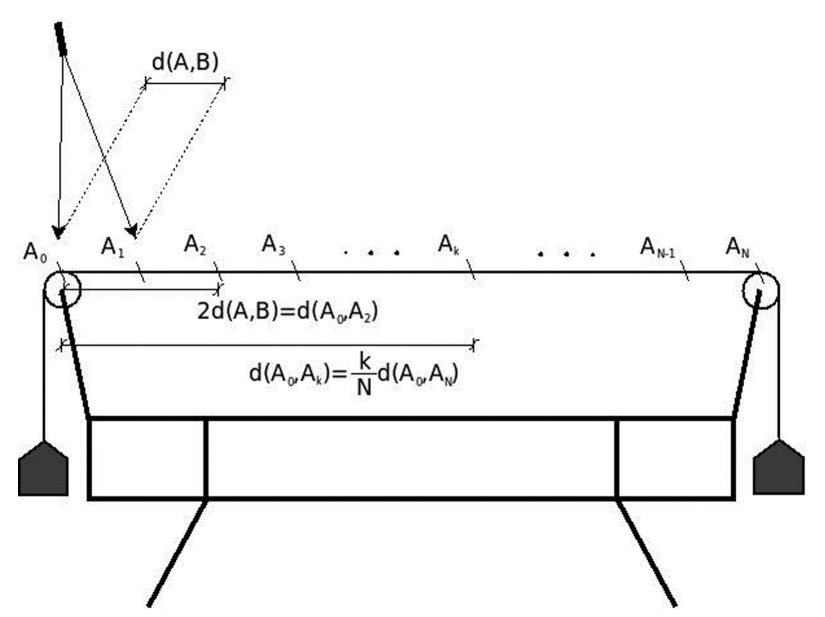

Figura 4: Construindo a multiplicação por escalar em $V_{L}$.

\footnotetext{
${ }^{3}$ Convidamos o leitor a propor um procedimento experimental que defina as noções de vertical e horizontal.
}

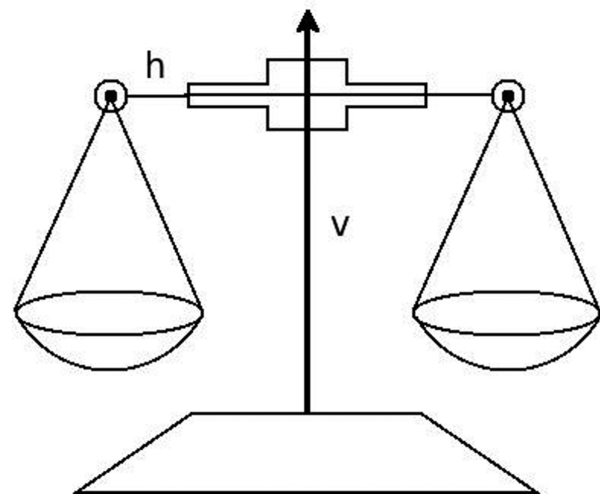

Figura 5: Balança de pratos para construção da grandeza massa.

$D_{M}$ é definido como o conjunto formado por objetos que, quando colocados em um dos pratos, causam um desequilíbrio na balança. Ressaltamos que este procedimento para construção $D_{M}$ é falho uma vez que se colocarmos uma formiga sobre um dos pratos, então provavelmente a balança não desequilibra. Contudo, em primeira aproximação, $D_{M}$ contém uma variedade grande de objetos do nosso mundo macroscópico. Diremos que dois objetos $a$ e $b$ em $D_{M}$ são (massa)-equivalentes quando ao colocá-los sobre os pratos, a balança permanece equilibrada. Neste caso, diremos que os valores $m_{a}$ e $m_{b}$ em $V_{M}$ são iguais. Também temos uma relação de ordem em $V_{M}$ : $m_{a}>m_{b}$, quando a balança pende para o lado do objeto a. A soma em $V_{M}$ é obtida ao colocarmos dois objetos $a$ e $b$ sobre o mesmo prato. A multiplicação por números é bastante intuitiva. Colocando $N_{1}$ objetos a iguais (massaequivalentes) sobre um prato, teremos o valor de massa $m=N_{1} m_{a}$. Vamos escrever o número $N_{1}$ como a soma de outros dois inteiros, digamos, $N_{1}=N_{2}+N_{3}$. Desta maneira, o produto por números $q$, racionais, pode ser visto ao retirarmos um número $N_{2}$ de massas $m_{a}$ da balança onde estavam as $N_{1}$ massas iniciais, restando uma massa $m^{\prime}=N_{3} m_{a}$. Comparando agora $m$ e $m^{\prime}$, chegamos a $m^{\prime}=q m$, com $q=N_{3} / N_{1}$. A extensão para reais positivos pode ser feita, por exemplo, depositando um líquido sobre o prato, completando assim os "buracos" deixados pelos racionais. Por fim, a multiplicação por negativos pode ser entendida como a retirada de massa da balança. Para fixar a base no espaço linear $V_{M}$, usamos um bloco padrão de certo material, que poderia, em princípio, ser reproduzido por qualquer pessoa, em qualquer lugar. Definiremos este bloco padrão como tendo o valor de $1 \mathrm{~kg}$. Observamos que apesar de depender da gravidade para que a balança seja defletida, a construção da grandeza massa não depende do local onde o procedimento é executado. Dois objetos massa-equivalentes na Terra também o são na Lua.

\subsection{Tempo: formalizando a clepsidra}

Quando construímos a grandeza distância, vimos que o procedimento experimental para obtenção das clas- 
ses $D_{L} / \sim$ exigia a noção de simultaneidade, ou seja, as duas pontas do compasso deveriam tocar os dois pontos de $D_{L}$ ao mesmo tempo. Utilizando esta construção como insight, vamos definir agora o que chamaremos de distância temporal, ou pelo menos no contexto deste trabalho, de intervalo de tempo ${ }^{4}$ A distância temporal terá como domínio $\left(D_{T}\right)$ o conjunto de pares de eventos. Um evento é um acontecimento localizado em uma região espacial tão pequena que pode ser considerada um ponto e com duração tão rápida que pode ser tratada como um instante 11. A cognição humana consegue diferenciar intervalos de tempo, mesmo sem uma definição formal para esta grandeza, que pretendemos construir. Por exemplo, mesmo sem uma medida, sabemos que é preciso esperar mais para enchermos uma garrafa de água do que um copo em uma mesma torneira. Mais ainda, abrindo e fechando a torneira com as mãos o mais rápido possível, é quase imperceptível o quanto esperamos entre o ato de abrir e fechar. Acontecimentos deste tipo fornecem a noção inicial de instante. Exemplos típicos de eventos são uma buzina de um carro, um flash ao tirarmos uma foto ou mesmo um piscar de olhos. Com a construção da grandeza intervalo de tempo, poderemos facilmente definir a noção de instante via limites, como é feito adiante; veja a expressão (18).

Agora construiremos operacionalmente as classes de equivalência no conjunto $D_{T}$ de pares de eventos. Para isto, usaremos um aparato bastante antigo e muito útil para os propósitos deste trabalho, a clepsidra [13]. O aparato consiste em um primeiro Reservatório (1), que despeja água no segundo (2). O Reservatório 2 tem um ladrão que mantém o nível da água constante. Uma torneira pode liberar a saída de água em 2. Tanto com a torneira aberta ou fechada o nível da água em 2 é mantido constante. Para isto, a vazão do reservatório 2 é menor do que a do 1, garantindo que está sempre entrando mais água em 2 do que saindo pela torneira e/ou ladrão. (Naturalmente, como o nível em 2 é mantido constante, a taxa líquida de saída de água é nula, uma vez que o que não sai pela torneira, acaba vazando pelo ladrão). Assim, quando o primeiro evento ocorre, a torneira é aberta e quando o segundo evento acontece, ela é fechada. Logo, medimos a massa de água fixando o que chamamos de intervalo de tempo ou distância temporal entre dois eventos. Aqui também usamos a noção de simultaneidade. A torneira é aberta (fechada) instantaneamente com o primeiro (segundo) evento. No domínio da mecânica clássica podemos assumir a troca de informações com velocidade infinita e logo, não há inconsistências com esta construção. Uma representação esquemática da clepsidra encontra-se na Fig. 6.

O uso da balança acaba por induzir tanto a soma como a multiplicação por números no espaço de valo-

\footnotetext{
${ }^{4} \mathrm{O}$ leitor familiar com a teoria da relatividade especial reconhece que o termo 'distância temporal' coincide, até certo ponto, com o que a literatura correspondente chama de tempo próprio. Em geral, tempo próprio e intervalo de tempo são diferentes [12].
}

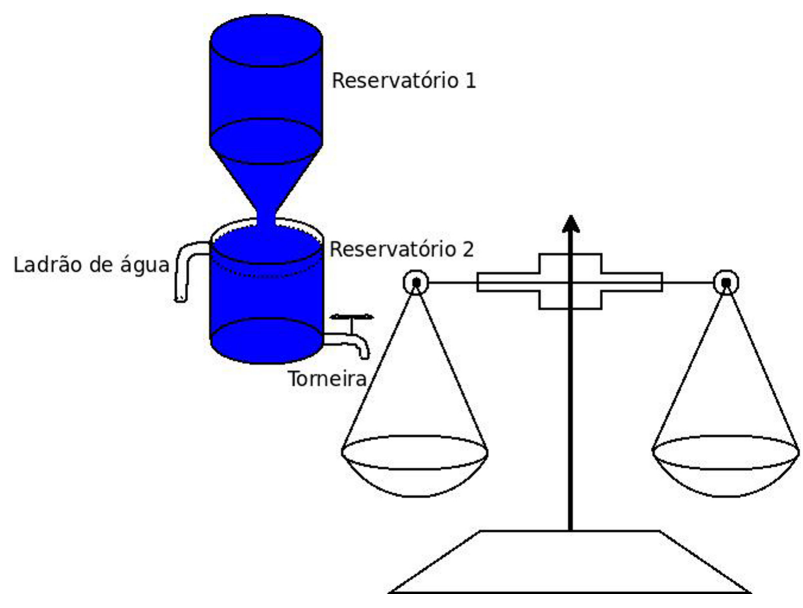

Figura 6: Representação esquemática da clepsidra associada a uma balança.

res $V_{T}$. Para dar um caráter mais universal ao aparato, poderíamos fixar, por exemplo, o volume de água no reservatório 2, o diâmetro da torneira, pressão atmosférica e temperatura da água. Contudo, o relógio depende explicitamente da gravidade local. O mero choque de um cometa com a Terra poderia alterar o funcionamento da clepsidra. Mas o propósito maior neste trabalho é caracterizar grandezas físicas e mesmo sem a devida precisão, foi possível construir o $D_{T}$, bem como o espaço $V_{T}$. A fixação de base em $V_{T}$ é feita a partir da medida de massa de água de algum evento periódico bem conhecido. Por exemplo, deixamos o Sol percorrer um arco de $45^{\circ}$ no céu e a massa de água medida corresponde, digamos, a 6 horas. Para completar esta Subseção, é possível dar uma definição precisa para instante. Basta tomar a sequência que assume valores em $V_{T}$,

$$
\begin{aligned}
s: & \mathbb{N} \rightarrow V_{T} \\
& n \mapsto s(n)=\frac{1}{n} \delta t,
\end{aligned}
$$

onde $\delta t$ é uma distância temporal tomada arbitrariamente. Chamaremos de instante o limite

$$
\lim _{n \rightarrow \infty} s(n) .
$$

Com isso, completamos a construção das três grandezas físicas que nos propomos a discutir neste artigo, L, M e T.

\section{Discussão geral sobre a construção de grandezas físicas}

Vamos estender um pouco da nossa discussão com uma lista de comentários.

\subsection{Grandezas primárias e secundárias}

Até aqui discutimos a construção de grandezas que podem ser obtidas a partir de um único procedimento experimental. Elas são chamadas de primárias. Se este não for 
o caso, então as grandezas são chamadas de secundárias. Um exemplo típico seria a rapidez de uma partícula em movimento unidimensional: dividimos a distância que ela percorre pelo intervalo de tempo gasto no percurso. Ou ainda, a área de uma figura plana é obtida pela multiplicação de duas distâncias.

\subsection{Produto e divisão de grandezas físicas}

$\mathrm{Na}$ discussão acima, mencionamos as palavras "multiplicação" e "divisão" de grandezas. Contudo, fizemos um esforço para mostrar que o conjunto de valores $V_{G}$ de uma grandeza $G$ é, na verdade, um espaço vetorial. As operações de multiplicação e divisão não estão definidas entre elementos de um espaço vetorial. O problema é ainda mais grave. Com efeito, toda a literatura escreve o momento linear de uma partícula como o produto $p=m v$, onde $m$ é a massa e $v$ a velocidade. Ora, o significado de $2 \times 4$ é claro. No fundo fazemos uma soma: $2 \times 4=4+4$. O que significa então $2 \mathrm{~kg}$ multiplicado por, digamos, $7 \mathrm{~m} / \mathrm{s}$ ? Vamos resolver estes dois conflitos. Consideremos duas grandezas $G$ e $H$ tais que $D_{G} \cap D_{H} \neq \varnothing$. V. g., para uma pessoa, é possível definir massa e altura. Gostaríamos de construir a grandeza $G H$. Sendo os espaços de valores $V_{G}$ e $V_{H}$ lineares, o espaço de valores de $G H$, cujo domínio é $D_{G} \cap D_{H}$, é definido pelo produto tensorial dos espaços

$$
V_{G H}=V_{G} \otimes V_{H} \text {. }
$$

Se dois objetos $O_{1}$ e $O_{2}$ possuem valores iguais das grandezas $G$ e $H$, isto é, $g_{1}=g_{2}$ e $h_{1}=h_{2}$, então exigiremos que $\left(v_{G H}\right)_{1}=\left(v_{G H}\right)_{2}$. Vamos usar a notação $v_{G H}=g \otimes h$. É razoável esperar que $\otimes$ tenha propriedades similares ao produto usual, isto é, seja distributivo tanto no primeiro quanto no segundo fator,

$$
\begin{array}{r}
\left(g_{1}+g_{2}\right) \otimes h=g_{1} \otimes h+g_{2} \otimes h \\
g \otimes\left(h_{1}+h_{2}\right)=g \otimes h_{1}+g \otimes h_{2},
\end{array}
$$

$\forall\left(g, g_{1}, g_{2} \in V_{G}\right)$ e $\forall\left(h, h_{1}, h_{2} \in V_{H}\right)$. Notemos que as somas do lado esquerdo das expressões 20 estão bem definidas. Já "+" do lado direito representa a soma em $V_{G H}$, que ainda não discutimos. Para isto, também é razoável pensar que dado $r \in \mathbb{R}$, então

$$
(r g) \otimes h=g \otimes(r h)
$$

De fato, considere uma colisão frontal de uma massa com valor $m$ e velocidade $2 v$ com outra massa $2 m$ e velocidade $v$. Supondo que as massas "grudem" ao se chocar, é um fato experimental que elas ficam paradas após a colisão. Este experimento simples fornece uma interpretação intuitiva para (22), levando certo conhecimento prévio de conservação de momento. Agora se quisermos escrever a soma de elementos $g_{1} \otimes h_{1}, g_{2} \otimes h_{2} \in V_{G H}$, buscamos $r \in \mathbb{R}$ tal que $g_{1}=r g_{2}$ e usamos 21] e 22

$$
\begin{aligned}
g_{1} \otimes h_{1}+g_{2} \otimes h_{2} & =\left(r g_{2}\right) \otimes h_{1}+g_{2} \otimes h_{2} \\
& =g_{2} \otimes\left(r h_{1}\right)+g_{2} \otimes h_{2}
\end{aligned}
$$

$$
=g_{2} \otimes\left(r h_{1}+h_{2}\right) \text {. }
$$

É um fato em Álgebra Linear que dados dois espaços vetoriais $U$ e $V$ de dimensão finita, temos

$$
\operatorname{dim}(U \otimes V)=\operatorname{dim} U \cdot \operatorname{dim} V
$$

Sendo ambos os espaços $V_{G}$ e $V_{H}$ unidimensionais, temos $\operatorname{dim} V_{G H}=1$. Vamos exibir uma base de $V_{G H}$. Dados vetores $v_{g} \in V_{G}$ e $v_{h} \in V_{H}$, os escrevemos como

$$
v_{g}=r \mathcal{U}_{G}, v_{h}=s \mathcal{U}_{H}
$$

onde $r, s \in \mathbb{R}$ e $\mathcal{U}_{G}, \mathcal{U}_{H}$ são as unidades (bases) de $G$ e $H\left(\right.$ dos espaços $V_{G}$ e $\left.V_{H}\right)$. Assim,

$$
\begin{aligned}
v_{g h} & =v_{g} \otimes v_{h} \\
& =\left(r \mathcal{U}_{G}\right) \otimes\left(s \mathcal{U}_{H}\right) \\
& =r s \mathcal{U}_{G} \otimes \mathcal{U}_{H} .
\end{aligned}
$$

A unidade da grandeza $G H$ é dada pela base $B=$ $\left\{\mathcal{U}_{G} \otimes \mathcal{U}_{H}\right\}$ de $V_{G H}$. Toda literatura conhecida ignora este fato e escreve simplesmente, por exemplo, $\mathrm{kg} \frac{\mathrm{m}}{\mathrm{s}}$ para momento linear no lugar de $\mathrm{kg} \otimes \frac{\mathrm{m}}{\mathrm{s}}$ e etc. Para o produto sucessivo da mesma grandeza, adota-se o expoente como notação. Por exemplo, $V_{\text {Área }}=V_{L} \otimes V_{L}$; uma base de $V_{\text {Area }}$ é dada por $\{m \otimes m\} \stackrel{\text { def. }}{=}\left\{m^{2}\right\}$. Para volume, teríamos $V_{\text {Vol. }}=V_{L} \otimes V_{L} \otimes V_{L}$. Neste caso, a base de $V_{V o l}$. é dada por $\{m \otimes m \otimes m\} \stackrel{\text { def. }}{=}\left\{m^{3}\right\}$. No caso geral, para uma grandeza $G$ arbitrária, define-se indutivamente o seu produto consecutivo, o espaço de valores e a base correspondente conforme a Tabela I.

Resta agora tratar a divisão de grandezas.

Sejam $G$ e $H$ grandezas físicas. Gostaríamos de definir $\frac{G}{H}$. À primeira vista, alguém desavisado gostaria de construir o espaço $V_{\frac{G}{H}}$ a partir da divisão de valores. Contudo, a divisão entre dois vetores não está definida; por outro lado, existem muitas grandezas com a forma $\frac{v_{g}}{v_{h}}$ com $v_{g} \in V_{G}$ e $v_{h} \in V_{G}$, como velocidade, aceleração, densidade, etc. Como corrigir este erro conceitual? A sugestão natural para tentarmos construir um análogo da divisão entre vetores é usar os espaços duais. Seja $(\mathcal{V}, \mathbb{R})$ um espaço vetorial sobre o corpo dos reais. Chamamos $\mathcal{V}^{*}$ o conjunto de todas as transformações lineares de $\mathcal{V}$ em $\mathbb{R}$. Com isto em mente, consideremos $H$ uma grandeza. Vamos construir a grandeza que será denotada por $H^{-1}$. O domínio de $H^{-1}$ será dado por

$$
D_{H^{-1}}=\left\{h \in D_{H} \mid v_{h} \neq 0 ; v_{h} \in V_{H}\right\} .
$$

Tabela 1: O produto de grandezas definido indutivamente.

\begin{tabular}{ccc}
\hline Grandeza & Espaço de valores & Base \\
\hline $\mathrm{G}$ & $V_{G}$ & $\left\{\mathcal{U}_{g}\right\}$ \\
$G G=G^{2}$ & $V_{G} \otimes V_{G}$ & $\left\{\mathcal{U}_{g} \otimes \mathcal{U}_{g}=\mathcal{U}_{g}^{2}\right\}$ \\
$\cdots$ & $\cdots$ & $\cdots$ \\
$G^{n} G \stackrel{\text { def. }}{=} G^{n+1}$ & $\underbrace{V_{G} \otimes \cdots \otimes V_{G}}_{n \text { vezes }} \otimes V_{G}$ & $\left\{\mathcal{U}_{g}^{n} \otimes \mathcal{U}_{g}=\mathcal{U}_{g}^{n+1}\right\}$ \\
\hline
\end{tabular}


O espaço de valores de $H^{-1}$ (com elementos que denotaremos por $v_{h}^{-1}$ ) é um subconjunto de $\mathcal{V}_{H}^{*}$, isto é, $v_{h}^{-1}: V_{H} \rightarrow \mathbb{R}$, com a seguinte restrição,

$$
\forall h \in D_{H^{-1}}, v_{h}^{-1}\left(v_{h}\right)=1 \in \mathbb{R} .
$$

Para grandezas unidimensionais que tratamos aqui, o mapeamento em (28) tem uma estrutura algébrica similar ao produto usual de números. Assim, dado $v_{h}=r \mathcal{U}_{h}$, com $r \in \mathbb{R}$ e $r \neq 0$, teremos $v_{h}^{-1}=\frac{1}{r} \mathcal{U}_{H}^{-1}$, sendo $\left\{\mathcal{U}_{H}^{-1}\right\}$ a base de $V_{H}^{-1}$. Como exemplo, considere a densidade linear $\mu$ de massa de uma corda. Esta grandeza assume valores no espaço $V_{M} \otimes V_{L}^{-1}$. A base deste espaço é dada por $k g \otimes m^{-1}$. Usualmente escreve-se $\frac{\mathrm{kg}}{\mathrm{m}}$. Um outro exemplo típico seria a rapidez de um móvel. Suponhamos que um automóvel percorre a distância $\delta l \in V_{L}$ entre duas cidades no intervalo de tempo $\delta t \in V_{T}$. A grandeza rapidez assume valores no espaço $V_{L} \otimes V_{T}^{-1}$, com base dada por $m \otimes s^{-1} \stackrel{\text { def. }}{=} m s^{-1}$. Definimos também indutivamente o produto para o inverso de grandezas. Para as grandezas $G, G^{2}, \ldots, G^{n+1}$, teremos a inversa, o espaço de valores e a respectiva base conforme a Tabela II.

Assim, concluímos nossa construção para o produto e divisão de grandezas físicas.

\subsection{Extensão dos domínios}

As prescrições adotadas aqui tanto para obtenção de $D_{G} / \sim$ quanto dos valores para distância, massa e tempo falham quando tentamos aplicá-las em regimes que fogem do nosso universo "palpável". Com efeito, você não colocará um compasso sobre dois átomos de um cristal para estimar a distância interatômica, não colocará também o Sol em uma balança e nem medirá a massa de água para estimar a duração de um ano. Contudo, as prescrições adotadas servem fortemente ao propósito do texto, uma vez que conseguimos construir toda a noção das grandezas básicas utilizadas na mecânica clássica. Naturalmente os valores das grandezas $\mathrm{L}, \mathrm{M}$ e $\mathrm{T}$ podem ser obtidos indiretamente para um domínio mais abrangente daquele discutido aqui.

\subsection{Valores de grandezas são vetores}

Depois de toda a discussão feita neste trabalho, faremos agora uma ressalva pedagógica. Devemos evitar escrever $M=1$ para o valor de uma certa massa, por exemplo. Isto é essencial principalmente para os estudantes dos anos iniciais de graduação. Números são diferentes de valores de grandezas físicas. Use sempre as unidades: $M=1 \mathrm{~kg}$. Ou você aceita algo do tipo $\vec{a}=2$ sem sentir algum desconforto?

\subsection{Incertezas de processos de medida}

Um fato bastante curioso, que aparece como corolário a partir da nossa postura operacional, é que números irracionais não podem ser obtidos/vistos em um laboratório. Se tentarmos medir a diagonal de um quadrado de $1 \mathrm{~cm}$ de lado com uma régua graduada em centímetros, obteríamos algo do tipo $l=1,41 \mathrm{~cm}$. Contudo, um estudante do ensino médio diria que a diagonal vale $\sqrt{2} \mathrm{~cm} \mathrm{e}$ é bem sabido que $\sqrt{2}$ é irracional, isto é, não pode ser escrito na forma de uma fração de números inteiros. Esta aparente contradição está ligada ao fato que qualquer procedimento experimental para determinação do valor de uma grandeza física carrega uma incerteza associada à medida.

Com o intuito de justificar a necessidade do conceito de incerteza associado a uma determinada medida, vejamos o seguinte exemplo. Um estudante pretende medir algumas vezes, utilizando a clepsidra já apresentada, a distância temporal entre o apagar e acender de uma lâmpada, que ocorre periodicamente devido ao circuito em que ela se encontra. O estudante acumula as quantidades de água, que representam os intervalos de tempo correspondentes. O fato curioso é que ao tentar colocar as massas de água, duas a duas, sobre a balança de dois braços, simplesmente há um desequilíbrio. De acordo com as regras de construção da grandeza física tempo, a distância temporal entre os eventos deveria ser a mesma para todas as medições, todavia, não é o que se verifica. Isso exige que ou descartemos a existência de um procedimento que associa um valor (numérico) à grandeza, o que representa uma atitude extrema que vai de encontro à descrição quantitativa da natureza, ou que sejamos um pouco mais brandos e consideremos a possibilidade de ocorrerem erros como consequência de algum detalhe intrínseco à medida, aparato ou de quem mede.

Partindo deste problema, seja uma grandeza física $G$ e um elemento $g$ de $D_{G}$. É necessário que tenhamos um valor teórico, que acreditamos ser o ideal para se medir o valor correspondente $v_{g}^{\text {T. }}$, que servirá como parâmetro para a medição de $v_{g}^{\mathrm{E}}$. Os sobrescritos T. e E. correspondem a 'Teórico' e 'Experimental'. Ao estimarmos o quanto $v_{g}^{\mathrm{E}}$ pode se diferenciar de $v_{g}^{\mathrm{T}}$, estamos lidando

Tabela 2: O produto de grandezas inversas.

\begin{tabular}{ccc}
\hline Inversa & Espaço de valores & Base \\
\hline$G^{-1}$ & $V_{G}^{-1}$ & $\left\{\mathcal{U}_{g}^{-1}\right\}$ \\
$\left(G^{2}\right)^{-1}$ & $V_{G}^{-1} \otimes V_{G}^{-1}$ & $\left\{\mathcal{U}_{g}^{-1} \otimes \mathcal{U}_{g}^{-1}=\mathcal{U}_{g}^{-2}\right\}$ \\
$\cdots$ & $\cdots$ & $\cdots$ \\
$\left(G^{n+1}\right)^{-1}$ & $\underbrace{V_{G}^{-1} \otimes \cdots \otimes V_{G}^{-1}}_{n \text { vezes }} \otimes V_{G}^{-1}$ & $\left\{\mathcal{U}_{g}^{-n} \otimes \mathcal{U}_{g}^{-1}=\mathcal{U}_{g}^{-(n+1)}\right\}$ \\
\hline
\end{tabular}


com um intervalo de valores, o qual é simétrico e contém $v_{g}^{\mathrm{T}}$ exatamente no centro, ou assim esperamos. Logo, uma medida do valor da grandeza $G$ para o objeto $g$ será escrita da seguinte maneira:

$$
v_{g}=\left(v_{g}^{\mathrm{E}} \pm \delta v_{g}\right) .
$$

$\delta v_{g}$ é o que chamaremos de incerteza experimental. Para uma grandeza que pode ser medida diretamente, $\delta v_{g}$ é fornecido por exemplo, pelo desvio-padrão de $v_{g}^{\mathrm{E}}$ calculado a partir de uma sequência de experimentos independentes e idênticos. É possível também obter a incerteza com uma medida só. Neste caso, $\delta v_{g}$ é dado pela incerteza do próprio aparelho de medida. Estas incertezas são chamadas de tipo A e B, respectivamente, e ainda é possível fazer uma combinação das duas 14.

Como consequência desta construção, diremos que o procedimento de medida da grandeza $G$ associada ao objeto $g$ foi satisfatório quando o módulo da diferença entre os valores teórico e experimental for menor que o valor da incerteza, ou seja,

$$
\left|v_{g}^{\mathrm{T} .}-v_{g}^{\mathrm{E} \cdot}\right|<\delta v_{g} .
$$

Além disso, esperamos também que a incerteza relativa definida por $u\left(v_{g}\right)=\delta v_{g} / v_{g}$ seja pequena. De fato, nos cursos de laboratório de Física Básica, espera-se $u\left(v_{g}\right)$ abaixo de 10\%. Se medirmos a espessura de uma folha de cartolina com um paquímetro, então o resultado seria algo como $(0,06 \pm 0,05) \mathrm{mm}$. Ora, a medida é da mesma ordem que a própria incerteza, o que torna o procedimento não mais confiável. Nosso próximo passo será lidar com incertezas associadas a valores de grandezas combinadas, como aquelas discutidas nos itens (A) e (B) acima. Consideremos inicialmente uma grandeza $H$ que depende do valor de uma, e somente uma, outra grandeza $G$. Por exemplo, a área de um quadrado depende da medida de um dos seus lados. Seja $F$ uma função que associa valores da grandeza $G$ a valores de $H$,

$$
\begin{aligned}
F: & V_{G} \rightarrow V_{H} \\
& v_{g} \mapsto F\left(v_{g}\right)=v_{h} .
\end{aligned}
$$

Assumindo que $F$ seja comportada, podemos estimar qual vai ser a incerteza de $v_{h}$ usando derivadas. Com efeito, dada uma função $f: \mathbb{R} \rightarrow \mathbb{R}$ derivável, podemos escrever

$$
f(x+\varepsilon)=f(x)+f^{\prime}(x) \varepsilon+o\left(\varepsilon^{2}\right),
$$

onde o erro $o\left(\varepsilon^{2}\right)$ vai a zero mais rápido que $\varepsilon$,

$$
\frac{o\left(\varepsilon^{2}\right)}{\varepsilon} \stackrel{\varepsilon \rightarrow 0}{\longrightarrow} 0
$$

Se desprezarmos termos de ordem superior $o\left(\varepsilon^{2}\right)$ em 32 assumindo que $\varepsilon \ll 1$, então ganhamos uma aplicação interessante das derivadas e fundamental à toda ciência moderna: fazer aproximações! Trocamos uma função qualquer nas proximidades do ponto $x$ por uma reta. Aplicando este resultado para a função $F$, calculada nas proximidades de uma medida dada por $v_{g} \pm \delta v_{g}$, ganhamos então a prescrição para obtenção da incerteza para $v_{h}$

$$
\delta v_{h}=\delta F=\left|F\left(v_{g}+\delta v_{g}\right)-F\left(v_{g}\right)\right|=\left|\frac{d F}{d v_{g}} \delta v_{g}\right|
$$

uma vez que esperamos de uma boa medida que $v_{g} \gg$ $\delta v_{g}$. O módulo acima garante que temos uma incerteza positiva, mesmo que a derivada seja negativa.

Consideremos agora uma grandeza $H$ cujos valore sejam dados por uma função de, digamos, $N$ valores de grandezas $G_{1}, \ldots, G_{N}$, isto é, um valor de $H$ para determinado objeto $h \in D_{H}$ é dado por $v_{h}=h\left(v_{1}, \ldots, v_{N}\right)$, com $v_{i} \in V_{G_{i}}, \forall i=1, \ldots, N$. Ou seja,

$$
\begin{aligned}
h: \prod_{i=1}^{N} V_{G_{i}} & \rightarrow V_{H} \\
\left(v_{1}, v_{2}, \ldots, v_{N}\right) & \mapsto h\left(v_{1}, v_{2}, \ldots, v_{N}\right)=v_{h} .
\end{aligned}
$$

Usamos a notação $\prod_{i=1}^{N} V_{G_{i}} \equiv V_{G_{1}} \times V_{G_{2}} \times \cdots \times V_{G_{N}}$. Vamos agora construir a incerteza associada a $v_{h}$. Suponhamos que a partir de certas medidas, foram obtidas as entradas da $N$-upla

$$
\bar{v}^{\mathrm{E} .}=\left(v_{1} \pm \delta v_{1}, \ldots, v_{N} \pm \delta v_{N}\right) .
$$

Usando a generalização da expressão 32 para o caso em que o domínio do mapeamento tenha mais variáveis do que uma,

$$
h\left(\bar{v}^{\mathrm{E} \cdot}\right)=h(\bar{v}+\delta \bar{v})=h(\bar{v})+d h[\delta \bar{v}] .
$$

Na expressão acima, fomos somente até primeira ordem nas incertezas e a diferencial de $h$ representada por $d h[\cdot]$ é um operador linear que leva elementos de $\prod_{i=1}^{N} V_{G_{i}}$ em $V_{H}$; dh é uma 1-forma 15], expressa por

$$
d h[\cdot]=\left.\sum_{i=1}^{N} \frac{\partial h}{\partial v_{i}}\right|_{\bar{v}} d v_{i}[\cdot] .
$$

Cada uma das diferenciais $d v_{i}[\cdot]$ 's atua da seguinte maneira: $d v_{i}(\bar{v})=v_{i}$. A expressão acima permite interpretar $d h[\cdot]$ como combinação linear de vetores básicos $\left\{d v_{1}, \ldots, d v_{N}\right\}$ e com coeficientes dados pelas derivadas parciais de $h$ estimadas em $\bar{v}$.

A origem para a incerteza de $v_{h}$ naturalmente reside nas incertezas $\delta v_{i}$ associadas a cada um dos valores de $v_{i}$, que por sua vez, estão ligados diretamente com a atuação de $d h[\cdot]$ em $\delta \bar{v}$. Definindo

$$
d h_{i}[\cdot]=\left.\frac{\partial h}{\partial v_{i}}\right|_{\bar{v}} d v_{i}[\cdot] \forall i=1, \ldots, N,
$$

nos resta combinar o que vamos chamar de incerteza para $v_{h}$ ao atuarmos com cada um dos $d h_{i}[\cdot]$ sobre as incertezas $\delta \bar{v}$. Uma possibilidade é tomar

$$
\delta v_{h}=d h[\delta \bar{v}]=\left.\sum_{i=1}^{N} \frac{\partial h}{\partial v_{i}}\right|_{\bar{v}} d v_{i}[\delta \bar{v}]=\left.\sum_{i=1}^{N} \frac{\partial h}{\partial v_{i}}\right|_{\bar{v}} \delta v_{i} .
$$


Este caso coincide com a nossa prescrição discutida acima quando a grandeza $H$ dependia somente de uma única variável. Uma outra possibilidade seria tomar, por exemplo,

$$
\delta v_{h}=\max _{i}\left\{\left.\frac{\partial h}{\partial v_{i}}\right|_{\bar{v}} \delta v_{i}\right\} .
$$

Esta alternativa, de certa forma, só leva em consideração a incerteza associada a uma das variáveis, a saber, aquela cujo valor da variação de h é máxima. Para sermos mais democráticos, vamos tomar como incerteza para $v_{h}$ a seguinte combinação

$$
\delta v_{h}=\sqrt{\left(d h_{1}[\delta \bar{v}]\right)^{2}+\cdots+\left(d h_{N}[\delta \bar{v}]\right)^{2}} .
$$

Desta maneira, levamos em consideração as incertezas associadas a variações do valor de $h$ quando todas as componentes $v_{i}$ são deslocadas pelas incertezas $\delta v_{i}$ correspondentes. Além disso, 42 tem uma interpretação geométrica clara. Construímos o espaço $\prod_{i=1}^{N} V_{H}$, isto é, o produto cartesiano de $N$ cópias de $V_{H}$. Cada um dos eixos corresponde aos valores que $v_{h}=h\left(v_{1}, \ldots, v_{N}\right)$ assume quando variamos alguma entrada $v_{i}$, mantendo as outras fixas. Isto pode gerar eixos formados por curvas, inclusive. Contudo, para aproximações lineares, podemos dotar este conjunto com uma estrutura euclidiana local no seguinte sentido: pequenas variações nas entradas $v_{i}$ devido às incertezas $\delta v_{i}$ geram deslocamentos dados por $d h_{i}[\delta \bar{v}]=\frac{\partial h}{\partial v_{i}} \delta v_{i}$, para $i=1, \ldots, N$. Assim, a expressão (42) que adotamos para a incerteza corresponde à diagonal de um paralelepípedo gerado por tais deslocamentos.

\subsection{Grandezas físicas multidimensionais}

Discutimos ao longo do texto como construir as grandezas L, M e T no domínio da mecânica clássica. Contudo, existem muitas outras grandezas de interesse que não são unidimensionais, incluindo, por exemplo, vetores e tensores. Grandezas multidimensionais serão tratadas em um trabalho futuro 16 .

\subsection{Um convite aos leitores}

Por fim, deixamos um questionamento a quem ler este artigo. Como seria possível construir a grandeza física temperatura? Qual o seu domínio? E a sua soma? É preciso defini-la a partir da troca de calor? E como ficam os reservatórios térmicos nesta história? Este exemplo ilustra o fato que, ao falarmos sobre qualquer grandeza que nos é usual, nem sempre conseguimos, de imediato, fornecer o seu domínio, espaço de valores e a soma correspondente.

\section{Conclusão}

Apresentamos ao longo deste artigo a noção de uma grandeza física G: começamos construindo o domínio $D_{G}$ da grandeza. Na sequência, obtivemos as classes de equivalência $D_{G} / \sim$ a partir de um procedimento experimental que caracteriza objetos $G$-equivalentes e, por fim, associamos aos objetos de $D_{G}$ valores em um conjunto $V_{G}$. Sobre $V_{G}$ define-se uma soma e uma multiplicação por números e com isso $V_{G}$ tem estrutura de espaço vetorial. Concluímos que as unidades da grandeza correspondem a uma base em $V_{G}$. Para elucidar toda a construção apresentada, descrevemos as grandezas distância, massa e tempo, centrais à mecânica clássica. Uma sequência de comentários foi feita na Seção III, que ilumina alguns pontos que em geral não são mencionados na literatura usual. Em particular, fornecemos também a multiplicação e divisão de grandezas a partir do produto tensorial entre espaços de valores e espaços duais, respectivamente, além de fornecer uma interpretação geométrica para a noção de incerteza experimental. Desta maneira, atingimos nosso objetivo central de complementar a definição de uma grandeza dada pelo JCGM.

Os autores adotaram ao longo do trabalho uma postura, de certa forma, operacional, defendida por P. W. Bridgman, que diz

$$
\begin{aligned}
& \text { it is a task for experiment to discover whether } \\
& \text { concepts so defined correspond to anything in } \\
& \text { nature, and we must always be prepared to find } \\
& \text { that the concepts correspond to nothing or only } \\
& \text { partially correspond. }
\end{aligned}
$$

Ressaltamos que uma abordagem puramente "operacionista" pode ser um tanto quanto controversa [17]. Contudo, nosso trabalho fornece uma possível maneira, diríamos, curiosa, para a apresentação da temática ligada a processos de medida e construção de grandezas físicas ${ }^{5}$

\section{Agradecimentos}

As noções discutidas neste trabalho foram tratadas em uma disciplina ministrada pelo Prof. Bernhard Lesche na Universidade Federal de Juiz de Fora, no segundo semestre de 2016. Os autores gostariam de agradecê-lo pelo empenho na formação de gerações de cientistas ao longo de sua carreira. Os autores são também gratos à turma de 'Física Contemporânea - 2017/03' do Mestrado Nacional Profissional em Ensino de Física - polo 24 - Juiz de Fora/MG pela leitura crítica e sugestões para a melhoria do texto. Este trabalho foi em parte financiado pelo Programa Institucional de Bolsas de Iniciação Científica - XXIX BIC/UFJF-2016/2017.

\section{Referências}

[1] P.W. Bridgman, Logic of Modern Physics (MacMillan, New York, 1927).

[2] Hermann von Helmholtz, Philosophische Aufsätze, Eduard Zeller zu seinem fünfzigjährigen Doctorjubiläum gewidmet (Fues Verlag, Leipzig, 1887), p. 17-52.

\footnotetext{
${ }^{5}$ Os autores gostariam de agradecer ao referee por apontar este ponto, enriquecendo o texto.
} 
[3] Otto L. Hölder, Mathematisch-Physikaliche Classe 53, 1 (1901). A tradução deste trabalho pode ser vista em Journal of Mathematical Psychology 40, 235 (1996).

[4] D.H. Krantz, R. Duncan, L.P. Suppes and A. Tversky, Foundations of Measurement, (San Diego, Academic Press, 1971), vols. 1-3.

[5] B. Lesche, Quantitative Sciences, disponível em <http://www.fisica.ufjf.br/ lesche/>, acesso em 04/03/2017.

[6] Joel Michell, Stud. Hisr. Phil. Sci. 24, 185 (1993).

[7] Joint Committee for Guides in Metrology, International Vocabulary of Basic and General Terms in Metrology - Basic and General Concepts and Associated Terms, disponível em https://www.bipm.org/utils/common/ documents/jcgm/JCGM_200_2012.pdf.

[8] Paul R. Halmos, Teoria Ingênua dos Conjuntos(Editora Ciência Moderna, Rio de Janeiro, 2001).

[9] Kenneth M Hoffman and Ray Kunze, Linear Algebra (Prentice-Hall, Englewood Cliffs, 1971).

[10] M. Alonso e E. Finn, Física - Um Curso Universitário, Vol. 1, Mecânica (Editora Edgar Blücher, São Paulo, 1972).

[11] B. Lesche, Teoria da Relatividade (Ed. Livraria da Física, São Paulo, 2005).

[12] V.A. Ugarov, Special Theory of Relativity (Mir Publishers, Moscow, 1979)

[13] Larry W. Mays (editor), Ancient Water Technologies (Springer, Dordrecht, 2010).

[14] INMETRO, Avaliação de Dados de Medição: Guia Para a Expressão de Incerteza de Medição - GUM 2008, disponível em <http://www.inmetro.gov.br/inovacao/ publicacoes/gum_final.pdf > acesso em 22/04/2017.

[15] Elon Lages Lima, Curso de Análise, vol. 2 (IMPA, Rio de Janeiro, 2005).

[16] Grandezas físicas multidimensionais. Em preparação.

[17] Hasok Chang, in: The Stanford Encyclopedia of Philosophy Edward N. Zalta (ed), disponível em <https://plato.stanford.edu/archives/fall2009/ entries/operationalism/>, acesso em 24/10/2017. 